\title{
PREVALENCIA DE SOBREPESO, OBESIDAD Y DISLIPIDEMIA EN TRABAJADORES DE SALUD DEL NIVEL PRIMARIO
}

\section{PREVALENCE OF OVERWEIGHT, OBESITY AND DYSLIPIDEMIA IN HEALTH WORKERS AT THE PRIMARY LEVEL}

\section{TITULO CORTO: PREVALENCIA DE SOBREPESO, OBESIDAD Y DISLIPIDEMIA}

\section{Gisela Gómez-Avellaneda ${ }^{1}$, Carolina Tarqui-Mamani ${ }^{2}$}

Recibido en octubre 11 de 2016

Aceptado en febrero 16 de 2017

Publicado en línea en mayo 15 de 2017

\section{Resumen}

La dislipidemia y la obesidad constituyen factores modificables de riesgo cardiovascular. El objetivo de este estudio es determinar la prevalencia de sobrepeso, obesidad y dislipidemia en trabajadores de la salud. Este es un estudio transversal en el que el muestreo fue aleatorio con afijación proporcional y se incluyeron 163 trabajadores de áreas de la salud. Se evaluaron el índice de masa corporal y el perímetro abdominal. Se consideraron colesterol total (CT) elevado > $200 \mathrm{mg} / \mathrm{dl}$, LDL-C > $100 \mathrm{mg} / \mathrm{dl}$, HDL-C bajo (varones HDL-C < $40 \mathrm{mg} / \mathrm{dl}$ y mujeres HDL-C < $50 \mathrm{mg} /$ dl), y triglicéridos $\geq 150 \mathrm{mg} / \mathrm{d}$. La prevalencia de hipercolesterolemia fue 30,1\%, triglicéridos 40,5\%, HDL bajo $69,3 \%$, LDL elevado $55,2 \%$, y la dislipidemia global fue $87,7 \%$. La mediana de triglicéridos ( $p=0,034$ ) y LDL-C fue mayor en varones que en mujeres $(p=0,038)$. La LDL-C aumentó con la edad $(p=0,015)$. La dislipidemia, sobrepeso, obesidad y obesidad abdominal predominaron en las mujeres. La prevalencia de sobrepeso fue $41,1 \%$, obesidad $25,8 \%$ y obesidad abdominal de $37,5 \%$. Se concluyó que la prevalencia de dislipidemia global fue alta y más de la mitad de los trabajadores de la salud presentaron sobrepeso u obesidad, así como la tercera parte tuvieron obesidad abdominal.

Palabras clave: hipercolesterolemia; hipertrigliceridemia; hiperlipidemias; dislipidemias; salud laboral.

\section{Abstract}

Dyslipidemia and obesity are modifiable risk factors of cardiovascular risk. To determine the prevalence of overweight, obesity and dyslipidemia in health care workers. Cross-sectional study was conducted. It carried out a sampling random with affixation proportional. The sample size was 163 health care workers. Body mass index and waist circumference anthropometric technique was evaluated. Considered high total cholesterol $>200 \mathrm{mg} / \mathrm{dl}$, high LDL-C > $100 \mathrm{mg} / \mathrm{dl}$, low HDL-C (men HDL-C $<40 \mathrm{mg} / \mathrm{dL}$ and women HDL-C $<50 \mathrm{mg} / \mathrm{dl}$ ), and triglycerides $\geq 150 \mathrm{md} / \mathrm{d}$. The

1. Nutricionista. Hospital Nacional Cayetano Heredia. Lima, Perú. Correo: gise_bugi@hotmail.com

2. Doctora en Salud Pública. Facultad de Medicina Humana, Universidad Nacional Mayor de San Marcos. Lima, Perú. Correo: carobtm@gmail.com 
prevalence of hypercholesterolemia was $30.1 \%$, 40.5\% triglycerides, $69.3 \%$ low HDL-C, high LDL-C 55.2\%, and global dyslipidemia was $87.7 \%$. The median triglycerides $(\mathrm{p}=0.034)$ and LDL-C was higher in males than in females $(p=0.038)$. The dyslipidemia increased as age increased in LDL-C $(p=0.015)$. Dyslipidemia, overweight, obesity and obesity abdominal were higher in workers women. The prevalence of overweight was $41.1 \%, 25.8 \%$ obesity and the obesity abdominal was $37.5 \%$. The prevalence of dyslipidemia global was high and more than half of health workers were overweight or obese and one-third had abdominal obesity.

Keywords: hypercholesterolemia; hypertriglyceridemia; hyperlipidemias; dyslipidemias; occupational health.

\section{INTRODUCCIÓN}

$\mathrm{S}^{\mathrm{e}}$ egún la Organización Mundial de la Salud (OMS) ${ }^{1}$, las enfermedades cardiovasculares (ECV) son la principal causa de muerte en el mundo. Se calcula que 17,5 millones de personas murieron por ECV. Aproximadamente, más de tres cuartas partes de las defunciones por ECV se producen en los países de ingresos bajos y medios. En Perú, la tercera causa de mortalidad son las enfermedades del aparato circulatorio ${ }^{2}$. Entre los factores de riesgo cardiovascular modificables están las dislipidemias, la hipertensión arterial, el tabaco, la diabetes mellitus, la vida sedentaria, el estrés, la obesidad y entre los no modificables están la edad, el sexo y la herencia.

En los últimos años la obesidad se ha duplicado a nivel mundial. Aproximadamente el $39 \%$ de la población adulta tenía sobrepeso y el $13 \%$ era obesa en los países con altos, medianos o bajos ingresos, sobre todo en la zona urbana ${ }^{3}$. Se estima que para el 2025 la prevalencia de obesidad será de $18 \%$ en los hombres y $21 \%$ en las mujeres adultas a nivel mundial ${ }^{4}$.

La dislipidemia es considerada como un factor de riesgo modificable de enfermedad coronaria y se define como la alteración de una o más lipoproteínas en sangre que conduce al aumento del colesterol total (CT), de triglicéridos (TG), al aumento de lipoproteínas de baja densidad (LDL-C) y a la disminución de las lipoproteínas de alta densidad (HDL-C) $)^{5}$. La dislipidemia es un factor de riesgo aterogénico con un efecto pronóstico que depende de la edad: cuanto más joven sea la persona, mayor será el impacto negativo sobre la esperanza de vida ${ }^{6}$.

En Perú, estudios poblacionales evidencian que la prevalencia de sobrepeso u obesidad asciende a 7,6\% en jóvenes y $19,8 \%$ en adultos ${ }^{7}$, mientras que la prevalencia de colesterol total es de 19,6\%, la hipertrigliceridemias de $15,0 \%$ y LDL-C elevado de $13 \%$ en adultos peruanos ${ }^{8}$. Los trabajadores de la salud no están exentos de este problema. Briceño-Díaz y Ayudant ${ }^{9}$ encontraron que el 48,8\% de los trabajadores de la salud del Hospital San José del Callao presentaron sobrepeso, $27,7 \%$ obesidad, y $53 \%$ tuvieron niveles de colesterol total por encima de lo recomendable $(<200 \mathrm{mg} / \mathrm{dL})$. Rosas et al10 mostraron que el 17,9\% de los trabajadores de una institución estatal tuvieron obesidad, 46,8\% sobrepeso, 34,7\% hipercolesterolemia, 19,5\% nivel alto de triglicéridos, 29,7\% LDL-C, 24,9\% de CT/HDL-C y 16,7\% de LDL-C/HDL-C.

El incremento de sobrepeso, obesidad y dislipidemia obedecen a factores genéticos y ambientales. Entre los factores ambientales tenemos el tipo de dieta, la baja actividad física, el bajo consumo de frutas y verduras, la ingesta de comida rápida con alto contenido de grasa y carbohidratos y bajo contenido de fibras, el consumo de bebidas azucaradas, entre otros ${ }^{6}$. La obesidad se asocia a niveles elevados de dislipidemias, este último incrementa el riesgo de enfermedades cardiovasculares y ambos afectan la expectativa y la calidad de vida de la persona, incrementan la muerte prematura ${ }^{11} \mathrm{y}$ repercuten en el Sistema de Salud debido al elevado costo por tratamiento y atención especializada ${ }^{12}$.

Los trabajadores de la salud constituyen una parte considerable del Sistema de Salud. Se requiere información sobre la situación de salud de este grupo laboral para proponer e implementar intervenciones preventivas para disminuir el exceso de peso y la dislipidemia, y así contribuir en la reducción del riesgo de enfermedades cardiovasculares.

El objetivo de este estudio fue determinar la prevalencia de sobrepeso, obesidad y dislipidemia en los trabajadores de salud en centros de salud de nivel primario. 


\section{MATERIALES Y MÉTODOS}

Este fue un estudio observacional y transversal. La población estuvo constituida por los trabajadores de la salud del nivel primario. Se incluyeron a los trabajadores de la salud con vínculo laboral y se excluyeron a los practicantes y trabajadores de la salud rotante o pasante. El muestreo fue aleatorio y con afijación proporcional. El tamaño de muestra se calculó considerando la proporción de triglicéridos de 48,6 \% en un total de población de 340 trabajadores de la salud; el nivel de confianza fue del $95 \%$ y la precisión del 5\%. La muestra calculada fue de 181 trabajadores de la salud distribuidos proporcionalmente entre los establecimientos del primer nivel de atención de la Red de Salud de Piedra Liza del distrito de San Juan de Lurigancho de Lima. Se evaluaron 163 trabajadores de la salud.

Se empleó la entrevista como técnica de recolección de datos y se aplicó un cuestionario adaptado, basado en el formato de la Encuesta Nacional de Indicadores Nutricionales, Bioquímicos, Socioeconómicos y Culturales Relacionados con las Enfermedades Crónicas Degenerativas, realizada por el Centro de Alimentación y Nutrición (CENAN) del Instituto Nacional de Salud ${ }^{8}$. El cuestionario incluyó los datos personales de antropometría y de dislipidemia.

Las mediciones del peso, talla y perímetro abdominal se realizaron según la norma técnica del Ministerio de Salud de Perú13 ${ }^{13}$ La evaluación nutricional se realizó mediante el Índice de Masa Corporal (IMC) que considera el peso/ talla al cuadrado. La clasificación del estado de nutrición se basó en los parámetros de la OMS y el Ministerio de Salud de Perú (MINSA). Para los adultos se consideró: delgadez (IMC $\leq 18,4)$, normal (IMC $\geq 18,5 \mathrm{y} \leq 24,9$ ), sobrepeso (IMC $\geq 25,0$ a $\leq 29,99$ ) y obesidad (IMC $\geq$ $30,0)^{14}$. y para los adultos mayores, delgadez (IMC $\leq 23,0$ ), normal (IMC $>23,0 \mathrm{y}<28,0)$, sobrepeso (IMC $\geq 28$ a $<$ $32,0)$, obesidad (IMC $\geq 32,00) 15$. Es necesario aclarar, que según norma técnica, en los adultos se considera obesidad I, II y III, pero en los adultos mayores, la clasificación incluye solamente obesidad, por ello, en el estudio, se consideró la obesidad en general.

La obesidad abdominal se obtuvo mediante el perímetro abdominal (PA) y se consideró en varones $\mathrm{PA} \geq 102 \mathrm{~cm}$ y mujeres $P A \geq 88 \mathrm{~cm})^{16}$. Es necesario precisar que el IMC evalúa el exceso de peso de la persona porque depende del peso y la estatura al cuadrado, pero no diferencia entre masa muscular y grasa; por tanto, podría sobreestimar o subestimar la obesidad sobre todo en atletas o en personas con menor masa corporal. Por otro lado, la obesidad abdominal identifica la grasa abdominal que está relacionada con el riesgo de padecer de enfermedades cardiovasculares.

Se determinó el perfil lipídico mediante el método colorimétrico en un laboratorio particular. Se extrajeron $5 \mathrm{ml}$ de sangre venosa pero previamente se le explicó al trabajador de la salud el procedimiento, la técnica y la importancia de que el trabajador estuviera en ayunas al menos durante ocho horas. Se consideraron colesterol total (CT) elevado > $200 \mathrm{mg} / \mathrm{dl}$, LDL-C de riesgo > 100 $\mathrm{mg} / \mathrm{dl}$, HDL-C alterado $<40 \mathrm{mg} / \mathrm{dl}$ en varones, HDL-C alterado $<50 \mathrm{mg} / \mathrm{dl}$ en mujeres, y triglicéridos $\geq 150$ $\mathrm{mg} / \mathrm{dl}$. Se le consideró dislipidemia a la presencia de al menos una dislipidemia ${ }^{16}$.

Se aplicó la prueba de Kolmogorov Smirnov para evaluar la normalidad de las variables cuantitativas. Ninguna variable tuvo distribución normal. Se calcularon medianas, percentil 25 y 75 para las variables cuantitativas y porcentajes para las variables cualitativas. Adicionalmente, se evalúo la diferencia de medianas mediante U Mann Whitney, diferencia de proporciones, chi cuadrado y razón de prevalencias. Se estableció como punto de corte para la significancia $(\mathrm{p}<0,05)$.

\section{Declaración sobre aspectos éticos}

Se solicitó el consentimiento informado por escrito de cada trabajador teniendo en cuenta la Declaración de Helsinki. Al finalizar la evaluación se entregaron los resultados en forma personal a cada participante. El proyecto fue aprobado por el Comité de Investigación y Ética de la Red de Salud de San Juan de Lurigancho de Lima, Perú.

\section{RESULTADOS}

Se evalúo a 163 trabajadores de la salud, el 75,5\% $(123 / 163)$ de los trabajadores fueron de sexo femenino, la mediana de edad fue 49,0 años y fluctúo entre 22 y 67 años; la mediana de la talla fue $156 \mathrm{~cm}$, peso $67,2 \mathrm{~kg}$, perímetro abdominal $88 \mathrm{~cm}$ y el IMC fue $27,5 \mathrm{~kg} / \mathrm{m} 2$.

Según el sexo, se observó que las medianas de triglicéridos $(p=0,0034)$, colesterol total $(p=0,052)$ y LDL-C $(p=0,081)$ fueron mayores en los hombres que en las mujeres, mientras que el HDL fue mayor en las mujeres $(p=0,038)$ (mayor información sobre el detalle de medianas se muestra en la Tabla 1). 
Tabla 1. Mediana de dislipidemia según sexo de trabajadores de salud en centros de salud de nivel primario.

\begin{tabular}{|l|c|c|c|c|c|c|c|}
\hline \multirow{2}{*}{ Dislipidemias } & \multicolumn{3}{|c|}{ Hombre (N=40) } & \multicolumn{3}{c|}{ Mujeres (N=123) } & \multirow{2}{*}{ Valor $\mathbf{p}^{*}$} \\
\cline { 2 - 7 } & Mediana & P25 & P75 & Mediana & P25 & P75 & \\
\hline Colesterol total & 187,4 & 161,0 & 218,0 & 174,7 & 161,1 & 207,0 & 0,052 \\
\hline Triglicéridos & 150,3 & 113,3 & 205,3 & 130,0 & 94,9 & 175,0 & 0,034 \\
\hline HDL & 37,9 & 32,5 & 48,5 & 43,0 & 35,9 & 53,1 & 0,038 \\
\hline LDL & 112,0 & 92,6 & 145,2 & 101,0 & 83,2 & 127,0 & 0,081 \\
\hline
\end{tabular}

$n=163$

*U Mann Whitney

La prevalencia global de dislipidemia fue de $87,7 \%$ (143/163) (IC 95\%: 82,4; 93,1), la hipercolesterolemia fue de $30,1 \%$ (IC 95\%:22,5; 37,4), la hipertrigliceridemia de 40,5\% (IC 95\%: 32,6; 48,3), el HDL bajo de 69,3\% (IC 95\%: 61,9; 76,7) y el LDL elevado $55,2 \%$ (IC 95\%: 42,3; 63,1), sin significancia estadística.

La prevalencia de hipertrigliceridemia aumentó conforme se incrementó la edad, a excepción de los adultos mayores $(\mathrm{p}=0,144)$. Situación similar se observó con la hipercolesterolemia $(p=0,096)$, LDL-C elevado $(p=0,015)$ y HDL-C bajo $(p=0,954)$. También se observó que las mujeres presentaron la mayor prevalencia de hipertrigliceridemia $(p=0,158)$, hipercolesterolemia $(\mathrm{p}=0,433)$, LDL-C elevado $(p=0,152)$ y DHL-C bajo $(p=0,281)$ que los varones. Esta información con mayor detalle se observa en la Tabla 2 y 3.

Tabla 2. Triglicérido y colesterol total según edad y sexo de los trabajadores de salud en centros de salud de nivel primario.

\begin{tabular}{|c|c|c|c|c|c|c|c|c|c|c|c|c|c|c|c|c|}
\hline \multirow{3}{*}{ Variables } & \multicolumn{8}{|c|}{ Triglicérido } & \multicolumn{8}{|c|}{ Colesterol total } \\
\hline & \multicolumn{2}{|c|}{$\mathrm{Si}$} & \multicolumn{2}{|c|}{ No } & \multirow{3}{*}{ Valor $p$} & \multirow{3}{*}{ RP } & \multirow{2}{*}{\multicolumn{2}{|c|}{ IC $95 \%$}} & \multicolumn{2}{|c|}{ Si } & \multicolumn{2}{|c|}{ No } & \multirow{3}{*}{ Valor $p$} & \multirow{3}{*}{$\mathrm{RP}$} & \multirow{2}{*}{\multicolumn{2}{|c|}{ IC 95\% }} \\
\hline & $\mathrm{N}^{0}$ & $\%$ & $\mathrm{~N}^{\mathrm{O}}$ & $\%$ & & & & & $\mathrm{~N}^{\mathrm{o}}$ & $\%$ & \begin{tabular}{|l|l}
$N^{\circ}$ \\
\end{tabular} & $\%$ & & & & \\
\hline \multicolumn{13}{|l|}{ Edad } & & & & \\
\hline 20 a 29 & 4 & 6,1 & 16 & 16,5 & 0,144 & 0,5 & $(0,2$ & $1,2)$ & 1 & 2,0 & 19 & 16,7 & 0,096 & 0,1 & $(0,0$ & 1.0) \\
\hline 30 a 39 & 10 & 15,2 & 22 & 22,7 & & 0,7 & $(0,4$ & $1,4)$ & 9 & 18,4 & 23 & 20,2 & & 0,8 & $(0,4$ & 1.7) \\
\hline 40 a 49 & 16 & 24,2 & 20 & 20,6 & & 1,0 & $(0,6$ & $1,8)$ & 14 & 28,6 & 22 & 19,3 & & 1,1 & $(0,6$; & 2.1) \\
\hline 50 a 59 & 25 & 37,9 & 25 & 25,8 & & 1,1 & $(0,7$ & $1,9)$ & 16 & 32,7 & 34 & 29,8 & & 0,9 & $(0,5$ & 1.7) \\
\hline 60 a 69 & 11 & 16,7 & 14 & 14,4 & & 1,0 & & & 9 & 18,4 & 16 & 14,0 & & 1,0 & & \\
\hline \multicolumn{17}{|l|}{ Sexo } \\
\hline Masculino & 20 & 30,3 & 20 & 20,6 & 0,158 & 1,3 & $(0,9 ;$ & $1,9)$ & 14 & 28,6 & 26 & 22,8 & 0,433 & 1,2 & $(0,7 ;$ & $2.0)$ \\
\hline Femenino & 46 & 69,7 & 77 & 79,4 & & 1,0 & & & 35 & 71,4 & 88 & 77,2 & & 1,0 & & \\
\hline
\end{tabular}

*Chi cuadrado

**Diferencia de proporciones

***RP: Razón de prevalencias 
Gisela Gómez-Avellaneda, Carolina Tarqui-Mamani

Tabla 3. LDL-C y HDL-C según sexo de trabajadores de salud en centros de salud de nivel primario.

\begin{tabular}{|c|c|c|c|c|c|c|c|c|c|c|c|c|c|c|c|c|}
\hline \multirow{4}{*}{$\begin{array}{l}\text { Variables } \\
\text { Edad }\end{array}$} & \multicolumn{8}{|c|}{ LDL-C } & \multicolumn{8}{|c|}{ HDL-C } \\
\hline & \multicolumn{2}{|c|}{$\mathrm{Si}$} & \multicolumn{2}{|c|}{ No } & \multirow{2}{*}{ Valor $\mathbf{p}$} & \multirow{3}{*}{$\mathbf{R P} * * *$} & \multirow{2}{*}{\multicolumn{2}{|c|}{ IC 95\% }} & \multicolumn{2}{|c|}{ Si } & \multicolumn{2}{|c|}{ No } & \multirow{3}{*}{ Valor $\mathrm{p}$} & \multirow{3}{*}{$\mathbf{R} \mathbf{P}^{* * *}$} & \multirow{2}{*}{\multicolumn{2}{|c|}{ IC 95\% }} \\
\hline & $\mathrm{N}^{\mathrm{o}}$ & $\%$ & $\mathrm{~N}^{0}$ & $\%$ & & & & & $\mathrm{~N}^{0}$ & $\%$ & $\mathrm{~N}^{0}$ & $\%$ & & & & \\
\hline & & & & & & & & & & & & & & & & \\
\hline 20 a 29 & 4 & 4,4 & 16 & 21,9 & $0,015^{*}$ & 0,4 & $(0,1 ;$ & $0,9)$ & 15 & 13,3 & 5 & 10,0 & $0,954^{*}$ & 1,1 & $(0,8$ & $1,6)$ \\
\hline 30 a 39 & 20 & 22,2 & 12 & 16,4 & & 1,2 & $(0,8$ & $1,9)$ & 23 & 20,4 & 9 & 18,0 & & 1,1 & $(0,7$ & $1,5)$ \\
\hline 40 a 49 & 23 & 25,6 & 13 & 17,8 & & 1,2 & $(0,8$ & $1,9)$ & 25 & 22,1 & 11 & 22,0 & & 1,0 & $(0,7$ & $1,4)$ \\
\hline 50 a 59 & 30 & 33,3 & 20 & 27,4 & & 1,2 & $(0,7$ & $1,8)$ & 33 & 29,2 & 17 & 34,0 & & 0,9 & $(0,7$ & $1,3)$ \\
\hline 60 a 69 & 13 & 14,4 & 12 & 16,4 & & 1,0 & & & 17 & 15,0 & 8 & 16,0 & & 1,0 & & \\
\hline Sexo & & & & & & & & & & & & & & & & \\
\hline Masculino & 26 & 28,9 & 14 & 19,2 & $0,152^{* *}$ & 1,2 & $(0,9 ;$ & $1,7)$ & 25 & 22,1 & 15 & 30,0 & $0,281^{* *}$ & 0,9 & $(0,7 ;$ & $1,1)$ \\
\hline Femenino & 64 & 71,1 & 59 & 80,8 & & 1,0 & & & 88 & 77,9 & 35 & 70,0 & & 1,0 & & \\
\hline
\end{tabular}

*Chi cuadrado

**Diferencia de proporciones

***RP: Razón de prevalencias

La prevalencia de sobrepeso fue de 41,1\% (67/163) (IC $95 \%: 33,2 ; 45,9)$ y la obesidad de $25,8 \%(42 / 163)$ (IC $95 \%: 18,7 ; 32,8)$, evidenciándose que ambos aumentaron a medida que se incrementó la edad (p $>0,05)$ y fueron más prevalentes en las mujeres ( $\mathrm{p}$
$>0,05$ ) (mayor detalle en la información se observa en la tabla 4). Adicionalmente, se observó que el grupo etario de 30 a 39 años tuvieron 2,2 veces la probabilidad de tener sobrepeso comparado con los adultos mayores.

Tabla 4. Sobrepeso, obesidad según edad y sexo de los trabajadores de salud en centros de salud de nivel primario.

\begin{tabular}{|c|c|c|c|c|c|c|c|c|c|c|c|c|c|c|c|c|}
\hline \multirow{3}{*}{ Variables } & \multicolumn{8}{|c|}{ Sobrepeso } & \multicolumn{8}{|c|}{ Obesidad } \\
\hline & \multicolumn{2}{|c|}{$\mathrm{Si}$} & \multicolumn{2}{|c|}{ No } & \multirow{2}{*}{ Valor $\mathbf{p}$} & \multirow{3}{*}{$\mathbf{R} \mathbf{P}^{* * *}$} & \multirow{2}{*}{\multicolumn{2}{|c|}{ IC 95\% }} & \multicolumn{2}{|c|}{ Si } & \multicolumn{2}{|c|}{ No } & \multirow{3}{*}{ Valor $p$} & \multirow{3}{*}{$\mathbf{R} \mathbf{P}^{* * *}$} & \multirow{2}{*}{\multicolumn{2}{|c|}{ IC 95\% }} \\
\hline & $\mathrm{N}^{0}$ & $\%$ & $\mathrm{~N}^{0}$ & $\%$ & & & & & $\mathrm{~N}^{0}$ & $\%$ & $\mathrm{~N}^{\mathrm{O}}$ & $\%$ & & & & \\
\hline \multicolumn{14}{|l|}{ Edad } & & & \\
\hline 20 a 29 & 9 & 13,43 & 11 & 11,5 & $0,203^{*}$ & 2,2 & $(0,9 ;$ & $5,6)$ & 2 & 4,8 & 18 & 14,9 & $0,122 *$ & 0,6 & $(0,1 ;$ & $3,1)$ \\
\hline 30 а 39 & 16 & 23,88 & 16 & 16,7 & & 2,5 & $(1,1 ;$ & $5,9)$ & 7 & 16,7 & 25 & 20,7 & & 1,4 & $(0,4$ & $4,1)$ \\
\hline 40 a 49 & 16 & 23,88 & 20 & 20,8 & & 2,2 & $(0,9 ;$ & $5,3)$ & 11 & 26,2 & 25 & 20,7 & & 1,9 & $(0,7$ & $5,3)$ \\
\hline 50 a 59 & 21 & 31,34 & 29 & 30,2 & & 2,1 & $(0,9 ;$ & 4,9) & 18 & 42,9 & 32 & 26,4 & & 2,2 & 0,8 & $5,9)$ \\
\hline 60 a 69 & 5 & 7,463 & 20 & 20,8 & & 1,0 & & & 4 & 9,5 & 21 & 17,4 & & 1,0 & & \\
\hline \multicolumn{17}{|l|}{ Sexo } \\
\hline Masculino & 22 & 32,8 & 18 & 18,8 & $0,04^{* *}$ & 1,5 & $(1,0$ & 2,2) & 9 & 21,4 & 31 & 25,6 & $0,587^{* *}$ & 0,8 & $(0,4$ & $1,6)$ \\
\hline Femenino & 45 & 67,2 & 78 & 81,3 & & 1,0 & & & 33 & 78,6 & 90 & 74,4 & & 1,0 & & \\
\hline
\end{tabular}

*Chi cuadrado

**Diferencia de proporciones

***RP: Razón de prevalencias 
En la Tabla 5 se observa que la prevalencia de obesidad abdominal fue de $37,5 \%$ (57/152) (IC $95 \%: 29,5 ; 45,5)$ y aumenta conforme se incrementa la edad $(p=0,189)$, siendo más frecuente en las mujeres $(\mathrm{p}=0,005)$.

Tabla 5. Obesidad abdominal según edad y sexo de los trabajadores de salud en centros de salud de nivel primario.

\begin{tabular}{|c|c|c|c|c|c|c|c|c|}
\hline \multirow{2}{*}{ Variables } & \multicolumn{2}{|c|}{$\mathrm{Si}$} & \multicolumn{2}{|c|}{ No } & \multirow{2}{*}{ Valor $p$} & \multirow{2}{*}{$\mathbf{R} \mathbf{P}^{* * *}$} & \multirow{2}{*}{\multicolumn{2}{|c|}{ IC $95 \%$}} \\
\hline & $\mathrm{N}^{0}$ & $\%$ & $\mathrm{~N}^{\mathrm{o}}$ & $\%$ & & & & \\
\hline \multicolumn{9}{|l|}{ Edad } \\
\hline 20 a 29 & 3 & 5,3 & 16 & 16,8 & $0,189 *$ & 0,4 & $(0,1 ;$ & $1,3)$ \\
\hline 30 а 39 & 10 & 17,5 & 21 & 22,1 & & 0,9 & $(0,4$ & $1,8)$ \\
\hline 40 a 49 & 14 & 24,6 & 19 & 20,0 & & 1,1 & $(0,6 ;$ & $2,2)$ \\
\hline 50 a 59 & 21 & 36,8 & 24 & 25,3 & & 1,2 & $(0,7 ;$ & $2,3)$ \\
\hline 60 a 69 & 9 & 15,8 & 15 & 15,8 & & 1,0 & & \\
\hline \multicolumn{9}{|l|}{ Sexo } \\
\hline Masculino & 7 & 12,3 & 31 & 32,6 & $0,005^{* *}$ & 0,4 & $(0,2 ;$ & $0,8)$ \\
\hline Femenino & 50 & 87,7 & 64 & 67,4 & & 1,0 & & \\
\hline
\end{tabular}

*Chi cuadrado

**Diferencia de proporciones

***RP: Razón de prevalencias

\section{DISCUSIÓN}

Los resultados encontrados muestran que uno de cada tres trabajadores de salud presentaron dislipidemias por hipercolesterolemia, siendo menor que lo reportado por Briceño-Díaz y Ayudant ${ }^{9}$ que evaluaron al personal que labora en un hospital del Callao. Situación similar se observó en los trabajadores de salud colombianos ${ }^{17} \mathrm{y}$ en adultos peruanos residentes en la zona altoandina de Perú ${ }^{18}$, ligeramente menor que lo resultados publicados por Orozco-González et a ${ }^{19}$ y similar a lo observado en adultos mexicanos ${ }^{20}$. Tres de cada siete trabajadores de salud padecieron de hipertrigliceridemia, siendo similar a lo reportado en los trabajadores colombianos ${ }^{17} \mathrm{y}$ trabajadores de salud mexicanos ${ }^{19}$, pero menor que lo observado en el personal de salud que labora en el hospital del Callao9. Una de las posibles explicaciones sería la elevada ingesta de carbohidratos, grasas saturadas ${ }^{21} \mathrm{y}$ la baja actividad física que se está incrementando en la población adulta ${ }^{22}$ situación de la que el personal de salud no estaría exenta.

En adición, se encontró que cinco de cada seis trabajadores de la salud presentaron algún tipo de dislipidemia. También se observó que dos de cada tres trabajadores de la salud presentaron HDL alterado, mayor que lo reportado en trabajadores de salud mexicanos ${ }^{19}$, siendo menor que lo reportado en adultos colombianos ${ }^{23}$ situación que es preocupante porque el incremento de los triglicéridos y el HDL alterado, aumenta el riesgo de morbimortalidad cardiovascular. Al respecto, en los últimos años se ha identificado un aumento mundial del consumo de comidas rápidas, alimentos altos en azúcares simples, harinas refinadas mezcladas con grasas vegetales saturadas, bajo consumo de frutas y verduras, y sedentarismo que, de cierta forma, podría explicar las elevadas prevalencias de las dislipidemias ${ }^{24}$. Merchant et a ${ }^{25}$ señaló que por cada cien gramos de carbohidrato se reduce $0,15 \mathrm{mmol} / \mathrm{L}$ de $\mathrm{HDL}^{25}$ y por cada uno por ciento de energía proveniente de grasas poliinsaturadas el HDL-C se reduce $0,023 \mathrm{mmol} / \mathrm{L}$ y aumenta los triglicéridos en $0,026 \mathrm{mmol} / \mathrm{L}^{25}$. De otro lado, algunos estudios muestran que los niveles altos de HDL tendrían un papel ateroprotector y antioxidante sobre el endotelio vascular porque propicia la peroxidación lipídica y favorece un ambiente vascular antitrombótico. Paralelamente, señala que el efecto del HDL sería menor que el $\mathrm{LDL}^{25,26}$.

Llama la atención que las dislipidemias son más predominantes en las mujeres que en los varones. Los resultados fueron coherentes con lo reportado en adultos chinos excepto en colesterol total ${ }^{27}$, situación que podría ser explicada porque los trabajadores de la salud generalmente son adultos y la mitad de las mujeres tienen 40 años o más y, probablemente, la mayoría en la etapa de la menopausia, que se caracteriza por el incremento de riesgo de dislipidemias en la mujeres debido a la disminución de los estrógenos ${ }^{26}$. De otro lado, 
se evidenció que la obesidad abdominal $(\mathrm{p}=0,005)$ y el sobrepeso fueron más frecuentes en las mujeres que en los hombres $(\mathrm{p}=0,04)$. Un hallazgo del estudio fue que el sexo masculino disminuye en $60 \%$ la probabilidad de obesidad abdominal comparada con las mujeres $(\mathrm{RP}=0,4$; IC $95 \%$ : 0,2; 0,8). Los resultados mostraron cierta similitud con lo reportado en trabajadores de un hospital del Callao ${ }^{9}$ pero menor que lo observado en trabajadores de la salud mexicanos ${ }^{19}$. Entre las posibles explicaciones se podría mencionar: la baja actividad física, el desplazamiento de los trabajadores a través de vehículos motorizados, el incremento de ingesta de comida rápida que se caracteriza por ser hipercalórica ${ }^{24}$, mayor tiempo del trabajo en oficina, el incremento del uso de computadoras para la labor administrativa que realizan los trabajadores de la salud, entre otros.

Una de las limitaciones del estudio es que se tuvo una tasa de no respuesta de aproximadamente $10 \%$ por diversas razones: ausencia de personal, negativa, y trabajadores de la salud que habían ingerido alimentos, lo que ameritó la exclusión de la muestra. Por otro lado, es importante considerar que los trabajadores de la salud han sido poco evaluados y la mayoría de estudios evalúan a los trabajadores en un solo establecimiento de salud u hospital, sin embargo, el presente estudio muestra la situación de dislipidemias en trabajadores de la salud de una microrred de salud que incluye ocho establecimientos de salud.

En conclusión, la prevalencia de dislipidemia global fue alta, la dislipidemia más frecuente fue el HDL bajo, la hipertrigliceridemia y la hipercolesterolemia, y más de la mitad de los trabajadores de salud presentaron sobrepeso u obesidad, así como la tercera parte de los trabajadores de la salud presentaron obesidad abdominal. Se sugiere a las autoridades del Ministerio de Salud implementar intervenciones educativas que sensibilicen y promuevan el bajo consumo de grasa saturadas de origen animal, azúcares procesados, la actividad física y la ingesta de agua diaria en los trabajadores de la salud. Asimismo, fomentar el consumo de aceite de oliva, frutas y verduras que tienen un efecto antioxidante y que previenen la aterosclerosis y la enfermedad cardiovascular ${ }^{28}$.

\section{DECLARACIÓN SOBRE CONFLICTOS DE INTERESES}

Los autores declaran no tener conflictos de interés y que el trabajo fue financiado por los investigadores.

\section{REFERENCIAS BIBLIOGRAFICAS}

1. Organización Mundial de la Salud. Factores de riesgo de enfermedades cardiovasculares: nuevas esferas de investigación. Informe de un Grupo Científico de la OMS. Ginebra: OMS; 1994.

2. Ministerio de Salud Dirección General de Epidemiologia. Análisis de situación de salud del Perú. [Internet]. Lima: DGE; 2013 [citado 22 Ene 2016]. Disponible en: http:// www.dge.gob.pe/portal/docs/intsan/asis2012.pdf.

3. Organización Mundial de la Salud [Internet]. Ginebra: Obesidad y sobrepeso [Actualizado Jun 2016; citado 14 Oct 2016]. Centro de prensa: Nota descriptiva $\mathrm{N}^{\circ} 311$ [Aprox. 7 pantallas]. Disponible en: http://www.who. int/mediacentre/factsheets/fs311/es/.

4. NCD Risk Factor Collaboration (NCD-RisC). Trends in adult body-mass index in 200 countries from 1975 to 2014: a pooled analysis of 1698 population-based measurement studies with 19.2 million participants. Lancet. 2016 April 2; 387:1377-96.

5. Canalizo-Miranda E, Eddie Favela-Pérez A, Salas-Anaya J, Gómez-Díaz R, Jara-Espino R, Torres-Arreola L, Viniegra-Osorio A. Guía de práctica clínica. Diagnóstico y tratamiento de las dislipidemias. Rev Med Inst Mex Seguro Soc. 2013;51(6):700-9.

6. Magallanes M, Gallegos E, Carrillo A, Sifuentes D, Olvera M. Sobrepeso, obesidad y dislipidemias en población universitaria del noreste de México. Rev Inv Educ en Enfermería. 2010; 28(1): 101-7.

7. Tarqui-Mamani C, Sánchez-Abanto J, Alvarez-Dongo D, Gómez-Guizado G, Valdivia-Zapana S. Tendencia del sobrepeso, obesidad y exceso de peso en el Perú. Rev Peru Epidemiol. 2013; 17(3):1-7.

8. Instituto Nacional de Salud. Encuesta Nacional de Indicadores Nutricionales, Bioquímicos, Socioeconómicos y Culturales Relacionados con las Enfermedades Crónicas Degenerativas. Lima: INS; 2006.

9. Briceño-Diaz R, Ayudant M. Estimación del riesgo cardiovascular mediante el sistema score en trabajadores de salud. Rev Med Rebagliti. 2013; 5(5):15-8.

10. Rosas A, Lama G, Llanos-Zavalaga F, Dunstan J. Prevalencia de obesidad e hipercolesterolemia en trabajadores de una institución estatal de Lima - Perú. Rev Perú Med Exp Salud Pública. 2002; 19 (2):87-92.

11. Ministerio de Salud de Chile. Informe Final Estudio de Carga de Enfermedad y Carga Atribuible, Chile; 2007.

12. Finkelstein EA, DiBonaventura M, Burgess SM, eta al. Thecosts of obesity in theworkplace. J Occup Environ Med 2010; 52 (10): 971-6.

13. Instituto nacional de salud (INS), Centro Nacional de Alimentación y Nutrición. La medición de la talla y el peso. Guía para el personal de salud del primer nivel de atención. Lima: INS; 2004. 
14. Instituto Nacional de Salud. Guía técnica para la valoración nutricional antropométrica de la persona adulta. Lima: INS; 2012.

15. Instituto Nacional de Salud. Guía técnica para la valoración nutricional antropométrica de la persona adulta mayor. Lima: INS; 2013.

16. National Cholesterol Education Program (NCEP) Expert Panel on Detection, Evaluation, and Treatment of High Blood Cholesterol in Adults (Adult Treatment Panel III).Third Report of the National Cholesterol Education Program (NCEP) Expert Panel on Detection, Evaluation, and Treatment of High Blood Cholesterol in Adults (Adult Treatment Panel III) final Report. Circulation. 2002 Sep; 106:3143-21.

17. Díaz-Realpe J, Muñoz-Martínez J, Sierra-Torres C. Factores de Riesgo para Enfermedad Cardiovascular en Trabajadores de una Institución Prestadora de Servicios de Salud, Colombia. Rev. Salud Pública. 2007; 9(1):64-75.

18. Málaga G, Zevallos-Palacios C, Lazo M, Huayanay C. Elevada frecuencia de dislipidemia y glucemia basal alterada en una población peruana de altura. Rev Perú Med Exp Salud Pública. 2010; 27(4): 557-61.

19. Orozco-González C, Cortés-Sanabria L, VieraFranco J, Ramírez-Márquez J, Cueto-Manzano A. Prevalencia de factores de riesgo cardiovascular en trabajadores de la salud. Rev Med Inst Mex Seguro Soc. 2016;54(5):594-601.

20. García-González I, Novelo-Ceh A, López-Novelo M, Ceballos-López A, Góngora-Bianchi R. Prevalencia de dislipidemias en población urbana aparentemente sana de Yucatán. Rev Latinoam Patol Clin Med Lab. 2015;62(3):150-6.

21. Latacunga D. Evaluación del consumo de alimentos y estado nutricional de la población adulta de Zamorano, Honduras
[Tesis de Ingeniero en Agroindustria Alimentaria]. Honduras: Escuela Agrícola Panamericana, El Zamorano; 2012. Disponible en: https://bdigital.zamorano.edu/ bitstream/11036/951/1/AGI-2012-T026.pdf.

22. Sanabria-Rojas H, Tarqui-Mamani C, Portugal-Benavides W, Pereyra-Zaldívar H, Mamani-Castillo L. Nivel de actividad física en los trabajadores de una Dirección Regional de Salud de Lima, Perú. Rev. Salud Pública. 2014;16(1): 53-62.

23. Galvis Y, Barona J, Cardona J. Prevalencia de dislipidemias en una institución prestadora de servicios de salud de Medellín (Colombia), 2013. Rev Medicina. 2016; 30(1):3-13.

24. Velasco-Contreras M. Perfil de salud de los trabajadores del Instituto Mexicano del Seguro Social. Rev Med Inst Mex Seguro Soc. 2013;51(1):12-25.

25. Merchant AT, Anand SS, Kelemen LE, Vuksan V, Jacobs $\mathrm{R}$, Davis B, et al. Carbohydrate intake and HDL in a multiethnic population. Am J Clin Nutr. 2007; 85:225-30.

26. Jeanine E, van Lennep R, Westerveld HT, WillemErkelens D, van der Wall E. Risk factors for coronary heart disease: implications of gender. Cardiovascular Research. 2002; 53 (3):538-49.

27. Qi L, Ding X, Tang W, Li Q, Mao D, Wang Y. Prevalence and Risk Factors Associated with Dyslipidemia in Chongqing, China. Int. J. Environ. Res. Public Health. 2015; 12(10):13455-65.

28. Díaz-Perilla M, Alarcón-Santamaría D, Amaya-Rodríguez $\mathrm{V}$. Efecto de un programa de atención nutricional sobre los valores del perfil lipídico de trabajadores de la Pontificia Universidad Javeriana con diagnóstico de dislipidemia. Rev facultad de Ciencias. 2005 Jul-Dic; $10 ; 71-80$.

Para citar este artículo: Gómez-Avellaneda G, Tarqui-Mamani C. Prevalencia de sobrepeso, obesidad y dislipidemia en trabajadores de salud del nivel primario. Duazary. 2017 julio; 14 (2): 141 - 148. Doi: http://dx.doi.org/10.21676/2389783X.1972 